J. Lake Sci. (湖泊科学), 2006, 18(6):649-654

http:// www. jlakes. org. E-mail: jlakes@ niglas. ac. cn

(c) 2006 by Journal of Lake Sciences

\title{
太湖螺类的实验生态学研究——以环棱螺为例”
}

白秀玲 ${ }^{1,2}$, 谷孝鸿 ${ }^{1 * *}$, 张 钰 ${ }^{1,2}$

( 1 : 中科院南京地理与湖泊研究所, 南京 210008 )

(2: 中国科学院研究生院, 北京 100039)

摘 要: 通过室内实验探讨环棱螺营养盐释放的影响因子及环棱螺对水体悬浮物、透明度及浮游植物的影响等生态特性. 结果表明: 温度及进食状况对环棱螺的营养盐释放均有显著影响. $15^{\circ} \mathrm{C}$ 时, 环棱螺氨氮的释放速率约为 $55.60 \pm 4.95 \mu \mathrm{g} /$ $(\mathrm{g} \cdot \mathrm{d})$, 磷酸根的释放速率约为 $2.073 \pm 0.120 \mu \mathrm{g} /(\mathrm{g} \cdot \mathrm{d})$; 而 $25^{\circ} \mathrm{C}$ 时, 环棱螺磷酸根的释放速率是 $2.819 \pm 0.075 \mu \mathrm{g} /(\mathrm{g}$ - d), 表明在一定温度范围内, 环棱螺营养盐的释放速率随温度升高而增加, 且进食状态下环棱螺的营养盐释放速率高于 饥饿状态. 此外, 环棱螺虽在短时间内可提高水体的透明度, 但其释放的营养盐可引起局部水体溶解态氮磷含量的增加, 在没有其它初级生产者争夺营养盐和光照等资源的情况下, 水体中藻类的再生速率会加快, 环棱螺难以对藻类的增殖起 到抑制作用. 由此建议在利用螺类进行生态调控的过程中, 必须与沉水植物的恢复紧密结合, 通过合理的空间搭配, 实现 水生态系统的恢复与健康发展.

关键词: 环棱螺;悬浮物质; 叶绿素 a; 太湖

\section{Studies on the experimental biology of snail in Lake Taihu: a case study of Bellamya spp.}

\author{
BAI Xiuling ${ }^{1,2}$, GU Xiaohong ${ }^{1} \&$ ZHANG Yu ${ }^{1,2}$ \\ (1: NanjingInstitute of Geography and Limnology, CAS, Najing 210008, P. R. China) \\ (2: Graduate School, CAS, Beijing 100039, P. R. China)
}

\begin{abstract}
To study the factors affecting the nutrient release of Bellamya sp., laboratory simulation experiments were carried out, and other traits of Bellamya sp. such as the effects of Bellamya spp. on suspended substance in the water column, water transparency and phytoplankton in the system were also investigated. Results showed that temperature and the amount of food had great influence on nutrient release of Bellamya sp. At $15^{\circ} \mathrm{C}$, the release rates of ammonia nitrogen and phosphate phosphorus by Bellamya spp. were $55.60( \pm 4.95) \mu \mathrm{g} /(\mathrm{g} \cdot \mathrm{d})$ and $2.073( \pm 0.1203) \mu \mathrm{g} /(\mathrm{g} \cdot \mathrm{d})$, respectively; at $25^{\circ} \mathrm{C}$, the release rate of phosphate phosphorus was about 2.819 $( \pm 0.075) \mu \mathrm{g} /(\mathrm{g} \cdot \mathrm{d})$. So the release rate of nutrient increased at an enhanced temperature , meanwhile nutrient release rate of snail eating food was higher than it that eating nothing. Bellamya sp. could increase the transparency of water on a short term, but the release of nutrients increased the contents of nitrogen and phosphorus in water, which in turn accelerated the regeneration of phytoplankton. Bellamya sp. can not control the production of algae completely, but their improving the secchi-disk depth of water is helpful for the growth and restoration of submerged macrophytes. It is suggested that the combination of biomanipulation of snails and restoration of submerged macrophytes will be an important technical way to control the eutrophication and improve water environment.
\end{abstract}

Keywords : Bellamya sp; suspended substance; Chl. a; Lake Taihu

在富营养湖泊的生态恢复与治理过程中, 通过引进大型软体动物种群来延缓富营养化进程、促进湖泊 生态恢复是较为常见的生态操纵措施之一 ${ }^{[1-3]}$. 目前, 对大型软体动物的研究主要集中在不同环境条件下

* 国家 “863”重大科技专项 (2002AA601011-04-02)、江苏省自然科学基金 (BK2002148) 共同资助. 2005 - $11-16$ 收稿;2006-05-21 收修改稿. 白秀玲,女, 1980 年生, 硕士研究生; E-mail : xlbai@ niglas. ac. cn.

** 通讯作者:谷孝鸿; E-mail:xhgu@ niglas. ac. cn. 
种类及生物量的变化, 而对其在物质循环和能量流动中的功能与作用研究较少 ${ }^{[1,4]}$. 在太湖的底栖动物组 成中, 大型软体动物螺、河蚬和蚌等在数量和生物量上均占有较高比例 ${ }^{[5]}$. 淡水瓣鳃类的蚌和蚬主要通过 滤食水体中的浮游藻类、有机碎屑等悬浮物质来改善水体环境, 如提高水体透明度、降低营养盐含量等,但 规格不同及湖水温度的变化等都对滤食能力有一定的影响 ${ }^{[6,7]}$; 腹足类螺的摄食类型是刮食, 目前, 有关螺 类对重金属的富集作用已有一定研究 ${ }^{[8]}$, 而对于螺类新陈代谢以及刮食行为对水体物质循环、理化环境等 方面的影响则报道较少 ${ }^{[9]}$. 本文以太湖常见的环棱螺为例, 通过室内实验探讨螺类的营养盐释放情况及其 对水体营养物质、悬浮物及浮游植物等的影响, 以期为富营养化水体的生物调控与治理提供参考依据.

\section{1 材料与方法}

\section{1 实验材料}

环棱螺取自太湖, 将其一部分暂养于种植沉水植物的整理箱内以备实验, 另一部分暂养于盛有曝气自 来水的容器中.

\section{2 实验设计}

1.2.1 不同状态下环棱螺营养盐的释放 选取放置时间 $48 \mathrm{~h}$ 以上的两种容器中的环棱螺各数个,称重之前 将其表层附着生物用软毛刷去除,清水洗净后, 用吸水纸将表层水分吸干.5 个螺 $(2.85-5.06 \mathrm{~g})$ 一组放于 $500 \mathrm{ml}$ 的三角瓶中, 以不放螺的作为参照, 每个三角瓶内注人实验用水 (自来水: 蒸馏水 =1:1) $400 \mathrm{ml}$, 两种 情况下均设 8 个平行样, 实验在光照培养箱中进行. 分别测量 $15^{\circ} \mathrm{C} 、 25^{\circ} \mathrm{C}$ 时两种情况下水体中的氨氮和磷酸 根的变化情况. 采样频率两小时一次,共采 6 次. 实验地点为太湖湖泊生态系统研究站.

1.2 .2 环棱螺对水体透明度及悬浮物的影响 实验用水取自太湖. 在实验组中, 将 10 个个体均匀且健康的 环棱螺放于 $2.5 \mathrm{~L}$ 的白色柱形有机玻璃缸内,且保证每个玻璃缸内螺的总重量接近,对照组不放螺,实验组 与对照组各设有两个平行样. 每两小时用移液管从玻璃缸中取水 $10 \mathrm{ml}$, 用紫外分光光度仪测定水样在 280 $\mathrm{nm}$ 至 $800 \mathrm{~nm}$ 的消光系数, 实验持续时间 $24 \mathrm{~h}$.

1.2.3. 环棱螺对浮游藻类的影响 实验设计同上. 实验组中, 7 个环棱螺一组, 个体大小均匀、健康, 且 保证每个玻璃缸内螺的总重量接近,对照组不放螺. 两组分别设有 12 个平行样,每次从各组随机抽取两个 平行样进行测定, 三天采样一次. 实验时间从 8 月 11 号至 8 月 29 号. 实验期间温度在 $26.5-32^{\circ} \mathrm{C}$ 之间.

\section{3 参数测定与方法}

选用 $0.45 \mathrm{~mm}$ 醋酸纤维微孔滤膜过滤水样, 然后测定溶解性总氮 (TDN)、溶解性总磷 (TDP)、氨氮 $\left(\mathrm{NH}_{4}^{+}-\mathrm{N}\right)$ 和磷酸根 $\left(\mathrm{PO}_{4}^{-}-\mathrm{P}\right)$, 未过滤的水样测定总氮 $(\mathrm{TN})$ 、总磷 $(\mathrm{TP})$. 总氮 $(\mathrm{TN})$ 、溶解性总氮 $(\mathrm{DTN}) 、$ 总磷 (TP) 、溶解性总磷 (DTP) 采用过硫酸钾消解法测定, 氨氮 $\left(\mathrm{NH}_{4}^{+}-\mathrm{N}\right)$ 采用纳氏试剂法测定, 磷酸根 $\left(\mathrm{PO}_{4}^{-}-\mathrm{P}\right)$ 采用钼锑抗比色法测定, 叶绿素 (Chl. a) 采用 $90 \%$ 的丙酮提取研磨后, 用苂光光度计测定. 悬移 质 $(\mathrm{SS}$ ) 用 Waterman GF/C 滤膜过滤后烘干, 用十万分之一的电子天平称量. 测定方法均按湖泊富营养调查 规范 ${ }^{[10]}$ 进行.

\section{2 结果与分析}

\section{1 不同状态下环棱螺释放氨氮与磷酸根的速率比较}

$15^{\circ} \mathrm{C}$ 时, 环棱螺在饥饿和进食两种状态下, 氨氮的释放速率不同. 在 $12 \mathrm{~h}$ 的实验期间,两种状态下前 $8 \mathrm{~h}$ 氨氮的释放速率都较为均匀,而后 $4 \mathrm{~h}$ 差异明显. 饥饿状态下环棱螺释放氨氮的速率较之前降低,而进食状 态下释放速率增加. 从平均速率分析, 饥饿状态下环棱螺释放氨氮的平均速率 $(48.0 \mu \mathrm{g} /(\mathrm{g} \cdot \mathrm{d}))$ 要明显小 于进食状态下释放的平均速率 $(63.2 \mu \mathrm{g} /(\mathrm{g} \cdot \mathrm{d})$ ) ( 图 1 a ). 两种状态下环棱螺释放磷酸根的速率较均匀, 进食状态下基本维持在 $2.316 \mu \mathrm{g} /(\mathrm{g} \cdot \mathrm{d})$ 左右, 饥饿状态下维持在 $1.836 \mu \mathrm{g} /(\mathrm{g} \cdot \mathrm{d})$ 左右 (图 $1 \mathrm{~b})$. 可见, $15^{\circ} \mathrm{C}$ 时环棱螺氨氮平均释放 $55.60 \pm 4.95 \mu \mathrm{g} /(\mathrm{g} \cdot \mathrm{d})$ 速率要明显高于磷酸根的平均释放速率 $2.073 \pm$ $0.120 \mu \mathrm{g} /(\mathrm{g} \cdot \mathrm{d})$.

$25^{\circ} \mathrm{C}$ 时, 进食状态下环棱螺释放磷酸根的速率较为均匀, 平均在 $2.964 \mu \mathrm{g} /(\mathrm{g} \cdot \mathrm{d})$. 饥饿状态下, 释放 磷酸根的速率略低于进食状态, 平均值为 $2.664 \mu \mathrm{g} /(\mathrm{g} \cdot \mathrm{d})$ ( 图 1 c c), 经计算, 该温度下环棱螺释放磷酸根 
的平均速率为 $2.819 \pm 0.075 \mu \mathrm{g} /(\mathrm{g} \cdot \mathrm{d})$. 上述分析可知,在同一温度下,饥饿状态下每克环棱螺营养盐的 释放速率均低于进食状态, 而在进食相同的情况下, 环棱螺的营养盐释放速率随温度升高而加快, 可见, 增 加食物源或适当升高温度均能在一定程度上促进环棱螺的新陈代谢.
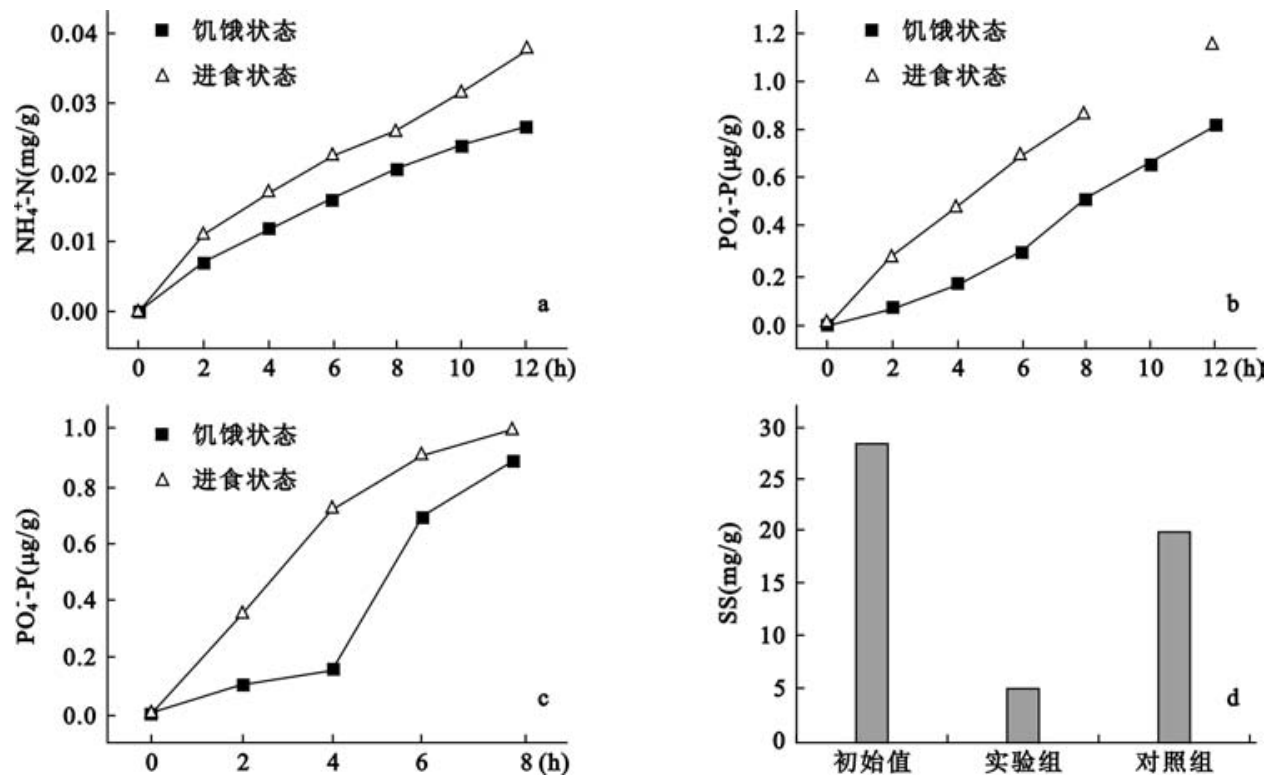

图 1 不同状态下环棱螺营养盐的释放及其对悬浮物质的影响

$\left(\mathrm{a}: 15^{\circ} \mathrm{C}\right.$ 下氨氮的释放情况; b: $15^{\circ} \mathrm{C}$ 下磷酸根的释放情况; $\mathrm{c}: 25^{\circ} \mathrm{C}$ 下磷酸根的释放情况; $\mathrm{d}$ : 环棱螺对悬浮物的影响 )

Fig. 1 The nutrient release of Bellamya sp. with the different states and the influence on suspended substance ( a: $15^{\circ} \mathrm{C}$, the release of $\mathrm{NH}_{4}^{+}-\mathrm{N} ; \mathrm{b}: 15^{\circ} \mathrm{C}$ the release of $\mathrm{PO}_{4}^{-}-\mathrm{P}$; c: $25^{\circ} \mathrm{C}$, the release of $\mathrm{PO}_{4}^{-}-\mathrm{P}$; d:the influence on suspended substance of Bellamya sp. )

\section{2 环棱螺作用下水体透明度的变化}

透明度是反映水中悬浮物质对透过光线阻碍程度大小的物理指标,水体中悬浮物质消光系数的变化反 映出水体透明度的变化. 经测定, 无论是实验组还是对照组, 水体的消光系数都随着波长的增加而减小. 在 相同波段下, 实验组水体的消光系数随着时间增加而逐步减小,在 $2 \mathrm{~h} 、 4 \mathrm{~h} 、 8 \mathrm{~h}$ 和 $24 \mathrm{~h}$ 时实验组水体每个波 段消光系数的均值分别为原样水体消光系数的 0.62 倍、 0.41 倍、 0.27 倍和 0.15 倍. 对照组水体的消光系 数随时间变化并不明显,在 $2 \mathrm{~h} 、 4 \mathrm{~h} 、 8 \mathrm{~h}$ 和 $24 \mathrm{~h}$ 时对照组水体每个波段消光系数的均值分别为原样水体消 光系数 1.14 倍、 0.73 倍、 1.00 倍和 0.80 倍, 实验结束时, 无螺对照组的消光系数要远高于有螺的实验组 (图 $2 \mathrm{a}, \mathrm{b}$ ). 从水体中悬浮物的变化情况来看, 初始水样中悬浮物 (SS) 浓度为 $28.20 \mathrm{mg} / \mathrm{L}, 24 \mathrm{~h}$ 后, 对照组中 由于悬浮物质的自然沉降, 悬浮物浓度有所下降, 平均值为 $19.82 \mathrm{mg} / \mathrm{L}$ (图 $1 \mathrm{~d}$ ), 而实验组中悬浮物变化非 常明显, 浓度值降低到 $4.98 \mathrm{mg} / \mathrm{L}$. 可见,水体中由于环棱螺的存在, 其悬浮物的含量出现大幅度的降低, 环 棱螺对水体悬浮物的这种消减作用一定程度上提高了水体的透明度,进而改善了水质.

\section{3 环棱螺作用下水体中浮游植物及营养盐的变化}

在淡水生态系统中, 底栖动物通过生物扰动及其对营养盐的吸收、转化、降解和排泄等生理活动, 影响 着水体营养盐含量的变化 ${ }^{[11]}$. 在实验水体生态系统中, 实验组水体氮磷营养盐含量均较初始值有不同程度 增加, 其总氮、溶解性总氮和氨氮含量分别为 $6.23 \mathrm{mg} / \mathrm{L} 、 4.00 \mathrm{mg} / \mathrm{L}$ 和 $2.69 \mathrm{mg} / \mathrm{L}$, 较水体氮含量初始值增 幅分别为 $125 \% 、 92 \%$ 和 $647 \%$, 总磷、溶解性总磷和磷酸根含量分别为 $0.415 \mathrm{mg} / \mathrm{L} 、 0.114 \mathrm{mg} / \mathrm{L}$ 和 0.038 


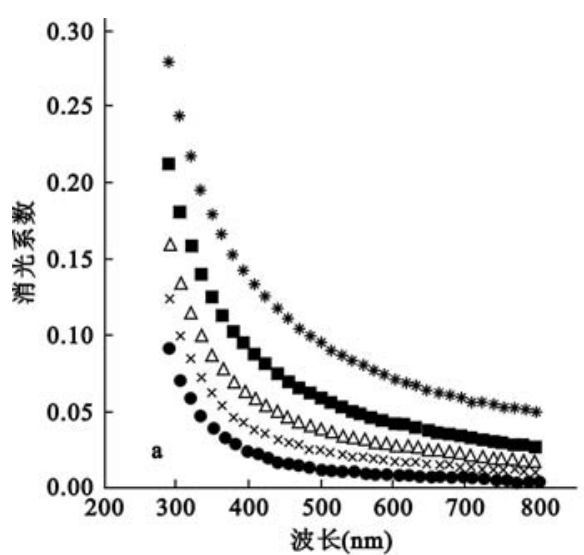

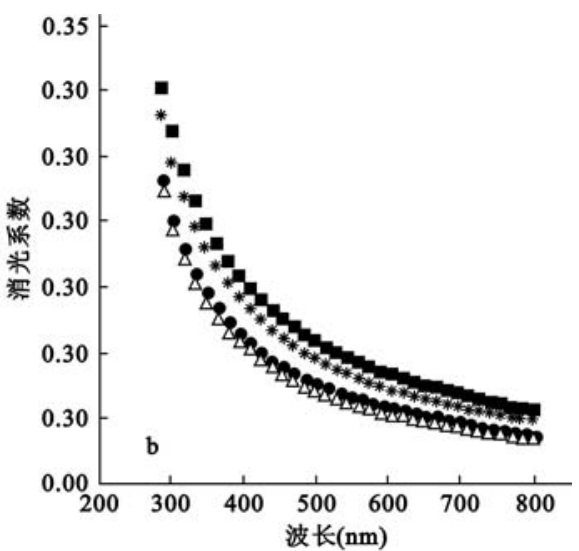

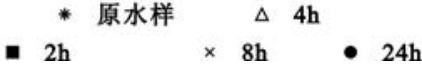

图 2 实验组和对照组水体在不同波段吸光度随时间的变化情况 ( $\mathrm{a}$ : 原样与实验组水体吸光度变化; $\mathrm{b}$ : 原样与对照组水体吸光度的变化)

Fig. 2 Comparison of the OD change of the water from $280 \mathrm{~nm}$ to $800 \mathrm{~nm}$ with the different conditions ( $a$ : between the original sample and the treatment; $b$ :between the original sample and the control)

$\mathrm{mg} / \mathrm{L}$, 较水体磷含量初始值增幅分别为 $80 \% 、 132 \%$ 和 $81 \%$. 而对照组水体营养盐含量与初始值相比,均有 不同程度的下降,各种形态氮、磷营养盐含量下降幅度均值分别为 $42 \%$ 和 $61 \%$ (表 1).

微型生态系统中,水体营养盐含量的变化影响着浮游植物生物量的变化. 由图 3 水体中藻类叶绿素 a 的变化趋势知,在 8 月 11 号 -8 月 14 号期间,实验组藻类叶绿素 a 的再生速率低于对照组,然随时间推移, 两组水体中藻类叶绿素 $\mathrm{a}$ 含量的变化情况完全不同,实验组中藻类叶绿素 $\mathrm{a}$ 的含量迅速增加,至实验结束 时, 其含量已达到初始值的 20 倍左右, 而对照组中叶绿素 $\mathrm{a}$ 的含量则逐渐减少, 实验结束时其含量仅为叶 绿素 a 初始值的 $1 / 4$.

表 1 实验前后水体营养盐的变化 (单位: $\mathrm{mg} / \mathrm{L}$ )

Tab. 1 Variation of water column nutrient during the experiment (unit: $\mathrm{mg} / \mathrm{L}$ )

\begin{tabular}{ccccccc}
\hline & $\mathrm{TN}$ & $\mathrm{DTN}$ & $\mathrm{NH}_{4}^{+}-\mathrm{N}$ & $\mathrm{TP}$ & $\mathrm{DTP}$ & $\mathrm{PO}_{4}^{-}-\mathrm{P}$ \\
\hline 初始值 & 2.76 & 2.08 & 0.36 & 0.231 & 0.049 & 0.021 \\
实验组 & 6.23 & 4.00 & 2.69 & 0.415 & 0.114 & 0.038 \\
对照组 & 1.60 & 0.96 & 0.25 & 0.084 & 0.019 & 0.009 \\
\hline
\end{tabular}

\section{3 讨论}

呼吸和排泄是生物体最基本而又重要的新陈代谢活动. 营养盐释放率的变化可直接反映其代谢活动的 强弱 ${ }^{[12]}$. 研究发现螺的新陈代谢强弱受多种因素制约, 已有实验证明, 个体较环棱螺稍小的 Lymnaea ( Galba ) turricula 释放磷酸根的速率为 $24.2 \pm 11.7 \mu \mathrm{g} /(\mathrm{g} \cdot \mathrm{d})^{[13]}$, 显然高于本研究中环棱螺释放磷酸根的速率, 这与软体动物个体大小和营养盐的释放速率呈反比的观点是相一致 ${ }^{[13]}$. 本实验结果显示, $15^{\circ} \mathrm{C}$ 下, 环棱螺 氨氮的释放速率约为 $55.60 \pm 4.95 \mu \mathrm{g} /(\mathrm{g} \cdot \mathrm{d})$, 磷酸根的释放速率约为 $2.073 \pm 0.120 \mu \mathrm{g} /(\mathrm{g} \cdot \mathrm{d})$. 而 $25^{\circ} \mathrm{C}$ 下, 环棱螺磷酸根的释放速率是 $2.819 \pm 0.075 \mu \mathrm{g} /(\mathrm{g} \cdot \mathrm{d})$, 这表明在一定温度范围内, 环棱螺营养盐的释 放速率随温度升高而增加. 当在同一温度情况下 $\left(15^{\circ} \mathrm{C}\right.$ 与 $25^{\circ} \mathrm{C}$ 时), 饥饿状态下环棱螺营养盐的释放率均低 于进食状态. 另外, 由于螺对不同食物的喜好程度不同, 在取食不同食物时, 其营养盐的释放速率亦 不同 ${ }^{[13]}$. 


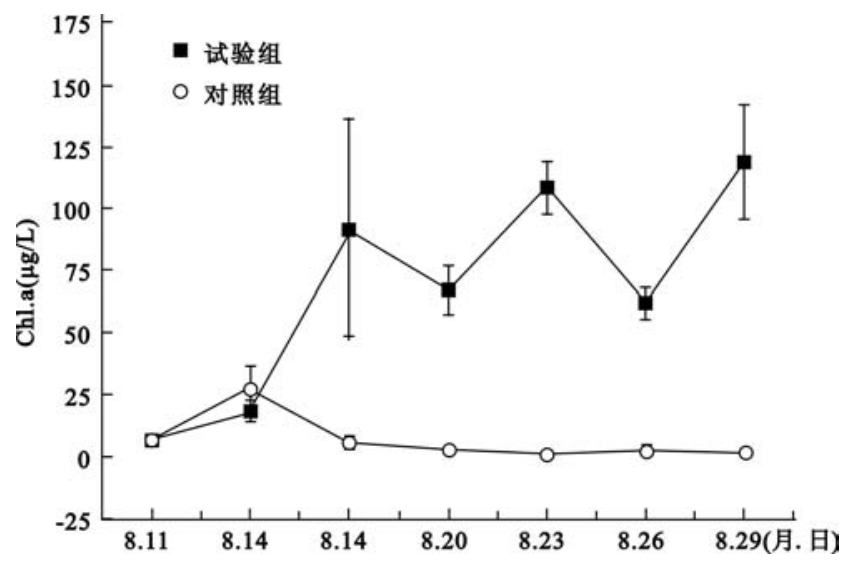

图 3 水体中藻类叶绿素 $\mathrm{a}$ 的变化趋势

Fig. 3 Vaviation of alga Chla in the waterbody

水体营养盐含量是衡量水质的一个重要指标. 本研究发现实验水体生态系统中, 实验组水体氮磷营养 盐含量较初始值明显增加,而对照组水体氮磷营养盐含量均不同程度的减少. 两组水体营养盐含量变化差 异的原因主要是: 环棱螺对水体营养盐的吸收、转化、降解和排泄等增加水体的营养盐含量, 同时设计的微 型生态系统水体是静止的而无法流动和交换,致使有螺的实验组水体营养盐不能得到稀释和有效利用, 这 些均促使实验组水体的营养盐含量大幅度增加; 而对照组水体营养盐含量下降主要在于水体中悬浮物质的 吸附和自然沉降, 同时水体中浮游植物光合作用吸收营养盐而生长繁殖, 随着时间推移, 藻类逐渐死亡沉积 于实验池底,而实验期间死亡的藻类并未被微生物及时分解, 从而使得水体营养盐含量出现下降.

透明度是衡量水质优劣的又一个重要指标. 实验组水体与对照组相比, 不论是水体悬浮物含量的变化, 还是水体消光系数随时间的变化,均表明环棱螺在短时间内可以提高水体的透明度. 因为环棱螺的分泌物 质可使水体中颗粒悬浮物迅速絮凝为团状,加快水体悬浮物质的沉降 ${ }^{[9]}$, 迅速减少水体悬浮物含量及降低 水体消光系数, 从而提高水体透明度.

环棱螺虽在短时间内可以提高水体透明度,改善水环境,但并不能完全抑制水体浮游藻类的增殖. 实验 结果表明: 在实验初期, 实验组中藻类的再生速率低于对照组,究其原因,主要是环棱螺分泌物质促使水体 中浮游植物絮凝沉降到容器底部, 同时环棱螺刮食也对藻类的增殖有一定的抑制作用. 但随时间的增加, 环 棱螺新陈代谢使得水体中营养盐含量逐渐增加,在没有其它初级生产者与藻类竞争营养盐和光照等资源的 情况下, 适宜的条件导致藻类的再生能力超过环棱螺对其生长的阻碍作用,从而引起藻类叶绿素 $\mathrm{a}$ 的含量 逐渐增加; 而对照组由于水体环境的变化, 在营养盐无法增加的情况下, 藻类自我死亡速率超过了其自生再 生速率,进而使得水体叶绿素 $\mathrm{a}$ 的含量出现下降趋势. 由此可推测仅靠放养环棱螺,难以抑制浮游藻类的增 殖. 同样底栖动物对水体浮游藻类的影响亦有实验发现: 背角无齿蚌高密度滤食可促进悬浮物絮凝沉降、有 效减少浮游藻类等悬浮物质、提高水体透明度等作用,对降低水体营养状况和富营养化水平没有明显 作用 ${ }^{[14]}$ 。

在水生态系统中,沉水植物和藻类同属于初级生产者,相互之间竞争营养盐、光照和生长空间等. 国内 外初步研究 ${ }^{[15-17]}$ 表明, 沉水植物对藻类的生长和暴发具有较好的抑制作用. 但在沉水植物的恢复过程中, 若藻类密度过高使得水体透明度过低,水生植物将难以在水生态系统中生存. 通过引进螺类等大型软体动 物可首先在一定程度上提高水体的透明度, 同时, 其代谢产物离子态的氮磷营养盐亦有利于水生植物的吸 收, 从而加快水生植物的恢复进程. 此外, 水生植物光合作用产生的氧气, 可满足大型软体动物生存之所需, 提高其成活率. 因此, 对沉水植物和螺类等软体动物进行空间合理配置和调控,在一定程度上可促进水生生 态系统的快速恢复. 


\section{4 参考文献}

[1] 刘保元, 邱东茹, 吴振斌. 富营养浅湖水生植被重建对底栖动物的影响. 应用与环境生物学报, 1997,3 (4) :323 - 327.

[2] Kairesalo T, Laine S, Luokkanen E, et al. Direct and indirect mechanisms behind successful biomanipulation. Hydrobiologia ,1999,395/396:99 - 106.

[3] Tuzun I, Mason C F, Eutrophication and its control by biomanipulation : an enclosure experiment. Hydrobiologia,1996,331 (1 - 3):79-85.

[4] 吴庆龙. 东太湖的贝类及其生物学. 海洋湖沼通报,1993,4:68-74.

[5] 孙顺才, 黄渏平主编. 太湖. 北京: 海洋出版社, 1993 .

[6] Reeders H H, Bij de Vaate A. Zebra mussels (Dreissena polymorpha) : a new perspective for water quality management. Hydrobiologia, 1990, 200/201:437 - 450.

[7] 王国祥. 富营养化湖泊生态修复的物理生态工程及其机理 [ 学位论文]. 南京: 中国科学院南京地理与 湖泊研究所, 1999 .

[8] 袁维佳,俞膺浩,谷 瑗等. 螺蛳对重金属元素的富集作用.上海师范大学学报(自然科学版), 2000, 29(3) : 73 - 79 .

[9] 魏阳春,兴培民. 太湖铜锈环棱螺对氮磷的降解作用. 长江流域资源与环境, 1999,8(1):88-93.

[10］金相灿,屠清瑛. 湖泊富营养化调查规范 . 北京:中国环境科学出版社, 1990 .

[11] Reise K. Tidal flat ecology : an experiment approach to species interaction. New York: Springer-verlag, 1985.

[12] 刘其根, 沈和定, 周洪琪等. 河蚬的耗氧率和排氨率. 上海水产大学学报, 1999,8 (4):298-303.

[13] Agnieszka Pinowska. Effects of snail grazing and nutrient release on growth of the macrophytes Ceratophyllum demersum and Elodea Canadensis and the filamentous green alga Cladophora sp. Hydrobiologia ,2002,479: 83 - 94 .

[14] 吴庆龙. 淡水双壳类的下行环境效应及其在环境调控中的应用一一以背角无齿蚌为例 [学位论文]. 南京: 中国科学院南京地理与湖泊研究所,2003.

[15] 陈开宁, 李文朝, 吴庆龙等. 滇池蓝藻对沉水植物生长的影响. 湖泊科学, 2003,15(4):364-368.

[16] Ozimek T, Pieczyska E,\& Hankiewicz A. Effects of filamentous algae on submerged macrophyte growth: a laboratory experiment. Aquatic Botany, 1991 ,41(4):309 - 315.

[17] 杨清心. 富营养水体中沉水植物与浮游藻类相互竞争的研究. 湖泊科学, 1996,8 (Suppl), $17-24$. 